\title{
LOCAL SYSTEMS OF LOCALLY SUPERSOLUBLE FINITARY GROUPS
}

\author{
C. J. E. PINNOCK* \\ School of Mathematical Sciences, Queen Mary, University of London, Mile End Road, London E1 4NS U.K. \\ e-mail: chris.pinnock@ukuug.org
}

(Received 21 January, 2002; accepted 20 August, 2002)

\begin{abstract}
We show that locally supersoluble finitary groups over certain division rings (e.g. fields) have local systems of hypercyclic normal subgroups.
\end{abstract}

2000 Mathematics Subject Classification. 20F19, 20 H99.

In 1960, M. S. Garaščuk [1] proved that a locally nilpotent linear group is hypercentral and in 1971, B. A. F. Wehrfritz extended this result by proving that a locally supersoluble linear group is hypercyclic ([7] Theorem B). A. E. Zalesskii investigated matrix groups over division rings in his 1969 paper [12]. One of the results of this paper was the following.

THEOREM 1 ([12]). Let $D$ be a locally finite-dimensional division algebra and let $G$ be a locally nilpotent matrix group over $D$. If $G$ is completely reducible or $D$ has characteristic zero, then $G$ is hypercentral.

Zalesskii leaves the general case (i.e. in positive characteristic when the group is not necessarily completely reducible) as a conjecture. Despite much effort, this conjecture has not been settled.

I. A. Stewart, in his Ph.D. thesis [5], generalized Zalesskii's result to locally supersoluble groups; that is, he showed that Theorem 1 holds with "nilpotent" replaced by "supersoluble" and "hypercentral" replaced by "hypercyclic".

A finitary skew linear group $G$ on the (left) vector space $V$ over the division ring $D$, is a subgroup of

$$
\mathrm{FGL}(V)=\left\{g \in \mathrm{GL}(V): \operatorname{dim}_{D} V(g-1)<\infty\right\} .
$$

We drop the word "skew" in the above if $D$ is a field. For an introduction to finitary linear groups, see [3].

We shall be interested in these groups only when $D$ is a locally finite-dimensional division algebra over a perfect field. We assume that $D$ satisfies this condition unless otherwise stated.

Now there exist locally nilpotent finitary linear groups which are as far away from being hypercentral as possible. For example, the McLain group $\mathrm{M}(\mathbb{Q}, \mathbb{C})$ (see $[\mathbf{1 0}]$ page 421) is locally nilpotent, finitary linear and has trivial hypercentre.

Wehrfritz has studied nilpotence in the finitary skew linear groups that we are interested in (see [10] and [9]). One of his results yields the following proposition, which

\footnotetext{
$\bar{*}$ This work was supported by an EPSRC grant whilst the author was at Queen Mary, University of London.
} 
says that locally nilpotent finitary groups, despite not necessarily being hypercentral, have a rich local structure of hypercentral normal subgroups.

PROPOSITION 2. Let $G$ be a locally nilpotent finitary skew linear group over $D$. Then $G$ has a local system of hypercentral normal subgroups. That is, if $X$ is a finite subset of $G$, then $\left\langle X^{G}\right\rangle$ is hypercentral.

Proof. For by [10] Corollary $1.3, G$ is generated by normal subgroups which are hypercentral of central height at most $\omega 2$.

In this note, we shall prove a generalization of Proposition 2:

THEOREM 3. Let $G$ be a locally-nilpotent by abelian subgroup of $\operatorname{FGL}(V)$. Then $G$ has a local system of hypercentral by finitely-generated-abelian normal subgroups.

Let $G$ be a locally supersoluble subgroup of $\operatorname{FGL}(V)$. Then any finite subset $X$ of $G$ lies in a hypercentral by finitely-generated abelian normal subgroup $H \triangleleft G$. Since $H$ is locally supersoluble, $H$ is hypercyclic by [8] Lemma 11.19. Consequently:

COROLlARY 4. Let $G$ be a locally supersoluble subgroup of FGL( $V)$. Then $G$ has a local system of hypercyclic normal subgroups.

We need some auxilary results. Note that with our assumptions on $D$, our results will include the general field case; for when $D$ is a field, we may suppose that it is algebraically closed. Also, with our hypotheses in Theorem 3, any subgroup of $G$ of $\mathrm{FGL}(V)$ has a maximal unipotent normal subgroup $\mathcal{U}(G)$ and any locally nilpotent subgroup $N$ of $\operatorname{FGL}(V)$ has a Jordan decomposition in $\mathrm{FGL}(V)$ into a unipotent part $N_{u}$ and a d-part $N_{d}$ (see [9] Lemma 2.8). The following lemma is a restatement of [9] 4.2(d).

LEMMA 5 (Wehrfritz). Let $G \leq \mathrm{FGL}(V)$ and let $N$ be a locally nilpotent normal subgroup of $G$ with $\mathcal{U}(N)=1$. For every finite subset $X$ of $G$, there is a normal subgroup $K$ of $G$ with $X \subseteq K$ and $N \cap K$ is hypercentral (of central height $\leq \omega 2$ ).

The Hirsch-Plotkin radical of the group $G$ is denoted by $\eta(G)$.

Lemma 6. Let $G \leq \mathrm{FGL}(V)$ and let $X$ be any subset of $G$ for which $n=\operatorname{dim}_{D}[V, X]$ is finite. Put $N=\eta\left(\left\langle X^{G}\right\rangle\right)$. Then $N_{u}$ is nilpotent of class $\leq 2 n$.

Proof. Let $\bar{G}=G\left(N_{d} \times N_{u}\right)$. Pick any $D-\bar{G}$ composition series of $V$, say $\left(V_{\alpha}, \Lambda_{\alpha}\right)_{\alpha \in I}$. Intersecting this series with $[V, X]$ and removing repititions, we obtain a finite series

$$
\begin{aligned}
0=[V, X] \cap V_{\alpha_{1}} & \leq[V, X] \cap \Lambda_{\alpha_{1}}=[V, X] \cap V_{\alpha_{2}} \leq \ldots \\
& \leq[V, X] \cap \Lambda_{\alpha_{i-1}}=[V, X] \cap V_{\alpha_{i}} \leq \ldots \\
& \leq[V, X] \cap \Lambda_{\alpha_{n}}=[V, X]
\end{aligned}
$$

where $\alpha_{1} \leq \alpha_{2} \leq \ldots \leq \alpha_{n}$ are elements of $I$.

Consider the series

$$
0 \leq V_{\alpha_{1}} \leq \Lambda_{\alpha_{1}} \leq V_{\alpha_{2}} \leq \ldots \leq V_{\alpha_{n}} \leq \Lambda_{\alpha_{n}} \leq V .
$$

Now $[V, X]=[V, X] \cap \Lambda_{\alpha_{n}}$ and $\left[V_{\alpha_{1}}, X\right] \leq[V, X] \cap V_{\alpha_{1}}=0$. Also if $1<i \leq n$ then

$$
\left[V_{\alpha_{i}}, X\right] \leq[V, X] \cap V_{\alpha_{i}}=[V, X] \cap \Lambda_{\alpha_{i-1}} \leq \Lambda_{\alpha_{i-1}} .
$$


Furthermore, the series 1 is $\bar{G}$-invariant and since $N \leq\left\langle X^{\bar{G}}\right\rangle$, we have $[V, N] \leq$ $\Lambda_{\alpha_{n}},\left[V_{\alpha_{1}}, N\right]=0$ and $\left[V_{\alpha_{i}}, N\right] \leq \Lambda_{\alpha_{i-1}}$ for $1<i \leq n$.

Let $B \leq A$ be $D-\bar{G}$ modules with $[A, N] \leq B$ and choose $n \in N$. On the factor $A / B$, we have $1 \equiv n \equiv n_{u} n_{d}$ as a Jordan decomposition for $n$. By the uniqueness of Jordan decomposition, $n_{u} \equiv 1$ on the factor $A / B$. In other words, $\left[A, N_{u}\right] \leq B$. Thus $\left[V, N_{u}\right] \leq \Lambda_{\alpha_{n}},\left[V_{\alpha_{1}}, N_{u}\right]=0$ and $\left[V_{\alpha_{i}}, N_{u}\right] \leq \Lambda_{\alpha_{i-1}}$ for $1<i \leq n$.

Put $C_{\alpha}=C_{\bar{G}}\left(\Lambda_{\alpha} / V_{\alpha}\right)$. Then $N_{u} C_{\alpha} / C_{\alpha}$ is a unipotent normal subgroup of the irreducible group $\bar{G} / C_{\alpha}$ for every $\alpha \in I$. By [11] 2.2, $N_{u} \leq C_{\alpha}$ and so $\left[\Lambda_{\alpha}, N_{u}\right] \leq V_{\alpha}$ for every $\alpha \in I$.

Consequently, $N_{u}$ stabilizes the series 1 and thus $N_{u}$ is nilpotent of class $\leq 2 n$ (for example, by [2] Theorem 1.C.1).

Proof of 3

Let $X$ be a finite subset of $G$ and put $H=\left\langle X^{G}\right\rangle$. Set $N=\eta(H)=H \cap \eta(G)$. Since $G / \eta(G)$ is abelian,

$$
H / N \cong H \eta(G) / \eta(G)=\langle\eta(G) x: x \in X\rangle .
$$

Thus $H / N$ is a finitely generated abelian group. Now it is sufficient to prove that $N$ is hypercentral.

There is an epimorphism $N \longrightarrow N_{d}$ with kernel $U=\mathcal{U}(N)$ (see [9] Lemma 2.8). Also $U \triangleleft G$; for $U^{g}$ is a unipotent normal subgroup of $N$ for every $g \in G$. Let $\bar{X}$ be the set $\{U x: x \in X\}$. Now $N / U$ is a locally nilpotent normal subgroup of $H / U$ with $\mathcal{U}(N / U)=1$ on its action on the sum of the composition factors of $V$ as a $D-G$ bimodule. Thus by 5 , there is $K \triangleleft G / U$ with $\bar{X} \subseteq K$ and $(N / U) \cap K$ hypercentral. Also $\left\langle\bar{X}^{G}\right\rangle=H / U$, so $N_{d} \cong N / U$ is hypercentral.

By $6, N_{u}$ is nilpotent and thus $N_{u} N_{d}$ is hypercentral (of height $\leq \omega 2$ ). Since $N \leq$ $N_{u} N_{d}$, we have that $N$ is hypercentral, as required.

To finish, we note the following result. It is a small extension of [6] Theorem 2.3.

Proposition 7. Let $G$ be a locally supersoluble finitary skew linear group over the division ring $D$, which is locally finite-dimensional over some (not necessarily perfect) subfield $F$. Then $G$ is locally-nilpotent by periodic-abelian.

Proof. Any finitely generated subgroup of $G$ is supersoluble and thus nilpotent by finite-abelian. The result follows using the local Zariski topology (for example, see [4] 2.2 Part 1).

\section{REFERENCES}

1. M. S. Garaščuk, On the theory of generalized nilpotent linear groups (Russian), Dokl. Akad. Nauk BSSR 4 (1960), 276-277.

2. O. H. Kegel and B. A. F. Wehrfritz, Locally finite groups (North-Holland, 1973).

3. R. E. Phillips, Finitary linear groups: A survey in Finite and locally finite groups (A. V. Borovik, R. M. Bryant, B. Hartley, and G. M. Seitz, eds.) (Kluwer Academic Publishers, 1995), 111-146.

4. C. J. E. Pinnock, Lawlessness and rank restrictions in certain finitary groups, Proc. Amer. Math. Soc. 130 (2002), 2815-2819. 1986).

5. I. A. Stewart, Locally supersoluble skew linear groups, Ph.D. thesis (Queen Mary College, 
6. I. A. Stewart, Supersolubility in certain skew linear groups, J. London Math. Soc. (2) 35 (1987), 91-97.

7. B. A. F. Wehrfritz, Supersoluble and locally supersoluble linear groups, J. Algebra 17 (1971), 41-58.

8. B. A. F. Wehrfritz, Infinite linear groups (Springer-Verlag, 1973).

9. B. A. F. Wehrfritz, Nilpotence in finitary skew linear groups, J. Pure and Applied Alg. 83 (1992), 27-41.

10. B. A. F. Wehrfritz, Nilpotence in finitary linear groups, Michigan Math. J. 40 (1993), 419-432.

11. B. A. F. Wehrfritz, Locally soluble finitary skew linear groups, J. Algebra 160 (1993), 226-241.

12. A. E. Zalesskii, The structure of several classes of matrix groups over a division ring, (Russian) Sibirsk. Math. Z̆. 8 (1967), 1284-1298. Translated in Siberian Math. J. 8 (1967), 978-988. 